\title{
Calibration of location errors of rotary axes on five-axis machine tools by on-the-machine measurement using a touch-trigger probe
}

\author{
$\operatorname{AUTHOR}(S)$ : \\ Ibaraki, Soichi; Iritani, Takeyuki; Matsushita, \\ Tetsuya
}

\section{CITATION:}

Ibaraki, Soichi ... [et al]. Calibration of location errors of rotary axes on five-axis machine tools by on-the-machine measurement using a touch-trigger probe. International Journal of Machine Tools and Manufacture 2012, 58: 44-53

\section{ISSUE DATE:}

2012-07

URL:

http://hdl.handle.net/2433/155455

\section{RIGHT:}

(C) 2012 Elsevier Ltd.; This is not the published version. Please cite only the published version.; この論文は出版社版でありません。引用の際に は出版社版をご確認ご利用ください。 


\title{
Calibration of location errors of rotary axes on five-axis machine tools by on-the-machine measurement using a touch-trigger probe
}

\author{
Soichi Ibaraki $^{\mathrm{a}}$, Takeyuki Iritani ${ }^{\mathrm{a}}$, Tetsuya Matsushita ${ }^{\mathrm{b}}$ \\ ${ }^{a}$ Department of Micro Engineering, Kyoto University, \\ Yoshida-honmachi, Sakyo-ku, Kyoto 606-8501, Japan. \\ Phone/Fax: +81-75-753-5227 \\ ${ }^{b}$ Okuma Corporation, Oguchi-cho, Niwa-gun, Aichi 480-0193, Japan.
}

\begin{abstract}
As a basis to improve the motion accuracy of five-axis machining centers, it is important to develop a methodology to calibrate location errors of rotary axes, one of fundamental error sources in the five-axis kinematics, in an accurate, efficient, and automated manner. This paper proposes a scheme to calibrate location errors of rotary axes by on-the-machine measurement of a test piece by using a contact-type touch-trigger probe installed on the machine's spindle. Compared to conventional calibration schemes described in latest revision of ISO standards (ISO/DIS 10791-1:2012 and ISO/DIS 107916:2012), where a precision sphere and a linear displacement sensor or the ball bar are used, the proposed approach is more suitable to efficient and automated calibration procedure of location errors, without requiring an experienced operator to perform the tests. The uncertainty analysis of the calibration of location errors is also presented with a particular interest in
\end{abstract}

Email address: ibaraki@prec.kyoto-u.ac.jp (Soichi Ibaraki) 
the influence of error motions of linear axes. The experimental demonstration is presented.

Keywords: Five-axis machine tools, rotary axis, location errors, touch-trigger probe, error calibration 


\section{Introduction}

Machine tools with two rotary axes to tilt and rotate a tool and/or a workpiece, in addition to three orthogonal linear axes, are collectively called five-axis machine tools. With an increasing need for machined components with geometric complexity in a high efficiency, they are extensively used in various manufacturing applications requiring higher machining accuracy. The improvement of their motion accuracies is a crucial demand in the market.

As a basis to improve the motion accuracy of five-axis machines, it is important to develop a methodology to measure it in an accurate, and efficient manner. ISO 10791-1 3 [1] standards contain quasi-static tests to calibrate static position and orientation errors of the axis average line of rotary axes. Lately, ISO TC39/SC2 has been revising ISO/DIS 10791-1:2012 [2] such that the tests can be also applied to five-axis machines with rotary axes in the workpiece side. Such errors are called location errors in ISO 230-7 [3], or geometric errors [4], link errors [5] in the literature. Numerical compensation of location errors of a rotary axis has been demonstrated by many researchers $[6,7,8]$. Some CNC makers lately commercialized an volumetric error compensation for rotary axes. Its implementation to mass-produced five-axis machines is, however, still limited. A clear issue is the lack of an efficient, and automated way to calibrate error motions in mass-production lines.

There has been many research works reported in the literature on the on-the-machine calibration of location errors of rotary axes $[9,10]$. The tests described in ISO/DIS 10791-1:2012 [2] use artefacts (e.g. a precision 
sphere or a straightedge) and a linear displacement sensor. The application of the telescoping double ball bar (DBB) has been also studied by many researchers $[4,11,12,13]$. Its inclusion in the revision of ISO/DIS 10791-6:2012 is currently under the discussion in ISO TC39/SC2 [14]. The full-automation of these measurement schemes is, however, difficult. Since one setup of ball bar measurement only measures the displacement in one direction, it requires at least a couple of different setups to identify all location errors. It requires an experienced operator to perform the tests. The R-test, presented by Weikert [15], Bringmann and Knapp [16], uses three (or four in [16]) displacement sensors to measure the three-dimensional displacement of the precision sphere (Zargarbashi and Mayer [5] recently presented an analogous instrument using non-contact capacitive sensors). The efficiency of the Rtest is a potentially significant advantage in the application to location errors calibration $[16,17]$. To use such a specialized measuring instrument for periodic check of the machine's accuracy in industrial applications, however, a user needs more experiences.

This paper proposes a scheme to calibrate all location errors of rotary axes by on-the-machine measurement of a test piece by using a contacttype touch-trigger probe installed on the machine's spindle. In recent years, high-accuracy touch-trigger probes for machine tools, which typically have one-directional measurement repeatability smaller than $1 \mu \mathrm{m}$, are available from some vendors. Many latest machining centers in the market by default come with a touch-trigger probe. ISO TC39/SC2 have been also discussing the standardization of test codes for measuring performance of such a touchtrigger probe (ISO/DIS 230-10 [18]). From its nature, a touch-trigger probe 
has a good communication capability with a CNC system, which potentially facilitates the automation of error calibration and compensation. Compared to conventional calibration schemes described in ISO/DIS 10791-1:2012 and ISO/DIS 10791-6:2012, the proposed approach is suitable to more efficient, and automated calibration procedure of location errors, without requiring an experienced operator to perform the tests.

To conduct routine checks of volumetric accuracy, the probing of mechanical artefacts such as ball arrays or ball plates has been widely studied particularly for CMMs [10, 19, 20]. Its application to machine tools has been studied in recent publications [21, 22]. The main focus of these approaches is on the calibration of volumetric errors of linear axes. Erkan et al. [22] presented the calibration of rotary axes in addition to linear axes, but only offset errors of the axis average line of rotary axes are considered. Some latest commercial CNCs have the capability to perform analogous probe-based error calibration. It is, to our knowledge, also limited to offset errors, which is only a part of rotary axis location errors.

\section{Error parameters to be identified and measuring instrument}

\subsection{Machine configuration}

This paper considers the 5 -axis machine configuration with a titling rotary table depicted in Fig. 1. The machine has three linear-axis drives (X, Y, and $\mathrm{Z}$ ) and two rotary-axis drives (A and $\mathrm{C}$ ). It must be emphasized that the basic idea of this paper can be straightforwardly extended to any configurations of five-axis machines. 


\subsection{Location errors to be identified}

Location errors define the position and the orientation of the axis average line of a rotary axis. ISO 230-7 [3] defines the axis average line as "a straight line segment located with respect to the reference coordinate axes representing the mean location of the axis of rotation." For the machine configuration depicted in Fig. 1, total eight location errors associated with A- and C-axes, shown in Table 1, are sufficient [23, 4].

The objective of this paper is to present a probe-based scheme to calibrate these eight location errors.

It is important to note that this paper assumes geometric errors of linear axes (X, Y, and Z-axes) are negligibly small compared to those of rotary axes. As was reviewed in Section 1, many five-axis error calibration methodologies have been recently studied (e.g. ball bar tests and the R-test). All of them only measure the relative displacement of the spindle tip to the table, and it is therefore not possible in principle to separate error motions of rotary axes and linear axes. To identify error motions of rotary axes, static error motions of linear axes are required to be separately pre-calibrated by conventional measurement (e.g. as shown in ISO 10791-1 [1]) and properly compensated as in [10]. The influence of linear axis error motions will be discussed in Section 5. 


\subsection{Kinematic Modeling of Five-axis Machine}

The kinematic model to compute the tool center position with respect to the workpiece is the basis of the error calibration presented in this paper. Although its derivation can be found in many previous publications [4, 17, 23], this subsection only briefly reviews it.

Define the machine coordinate system as the coordinate system fixed to the Y-axis frame. Suppose that the tool center location in the machine coordinate system is given by ${ }^{r} q \in \mathbb{R}^{3}$. The left-side superscript $r$ represents a vector in the machine coordinate system.

Define the workpiece coordinate system as the coordinate system attached to the rotary table. The homogeneous transformation matrix (HTM) representing the transformation from the workpiece coordinate system to the reference coordinate system is given by:

$$
\begin{aligned}
{ }^{r} T_{w}= & { }^{y} T_{a}{ }^{a} T_{c} \\
{ }^{a} T_{c}= & D_{y}\left(\delta y_{C A}\right) D_{a}\left(\alpha_{C A}\right) D_{b}\left(\beta_{C A}\right) D_{c}\left(\gamma_{C A}\right) D_{c}\left(-C^{*}\right) \\
{ }^{y} T_{a}= & D_{x}\left(\delta x_{A Y}\right) D_{y}\left(\delta y_{A Y}\right) D_{z}\left(\delta z_{A Y}\right) \\
& D_{a}\left(\alpha_{A Y}\right) D_{b}\left(\beta_{A Y}\right) D_{c}\left(\gamma_{A Y}\right) D_{a}\left(-A^{*}\right)
\end{aligned}
$$

where $D_{x}(x), D_{y}(y)$, and $D_{z}(z) \in \mathbb{R}^{4 \times 4}$ represent the HTM for linear motions in X-, Y-, and Z-directions. $D_{a}(a), D_{b}(b)$, and $D_{c}(c) \in \mathbb{R}^{4 \times 4}$ represent the HTM for angular motions about X, Y and Z axes. See e.g. [4, 23] for their formulation. $A^{*}$ and $C^{*} \in \mathbb{R}$ represent the command angular position of $\mathrm{A}$ and $\mathrm{C}$ axes, respectively.

Hence, the tool center location in the workpiece coordinate system, ${ }^{w} q \in$ 
$\mathbb{R}^{3}$, is given by:

$$
\left[\begin{array}{c}
{ }^{w} q \\
1
\end{array}\right]=\left({ }^{r} T_{w}\right)^{-1} \cdot\left[\begin{array}{c}
{ }^{r} q \\
1
\end{array}\right]
$$

The left-side superscript $w$ denotes the vector defined in the workpiece coordinate system.

\subsection{Measuring Instrument}

This paper uses a typical contact-type touch-trigger probe for discretepoint probing. The probe approaches to the object surface in the direction normal to it. When the contact of a probe ball with the object is detected, a signal is sent to a CNC to stop the drive and record its position in the machine coordinate system. The position of the contact point on the surface is calculated from the machine position, the approaching direction and the calibrated ball radius. According to the probe software's standard procedure (or [18]), the pre-travel variation for different approaching directions must be compensated.

\section{Probing procedure and identification of location errors}

\subsection{Probing Patterns}

In [24], a part of the authors presented a set of cutting test patterns to identify all location errors by measuring geometric errors of the machined test piece. Probing patterns presented in this section can be understood as a straightforward translation of these cutting tests.

Figures 2(a) to (h) schematically depict proposed probing patterns. As 
an example, Probing pattern 2-a (in Fig. 2(b)) will be described in details. The rotary table is placed horizontally by the A-axis $\left(A=0^{\circ}\right)$. Place a test piece of a square column geometry at the $+X$ location as shown in (i). Then, perform a standard probing procedure to measure the $\mathrm{X}$ position of the target point from the $+X$ direction. Denote this $\mathrm{X}$ position in the machine coordinate system by $x_{1}^{2 a}>0$. Then, rotate the rotary table by $C=180^{\circ}$ as shown in (ii), and probe the same target point from $-X$ direction (denote measured position by $\left.x_{2}^{2 a}<0\right)$. The origin of the machine coordinate system is set at the intersection of nominal C- and A-axes.

In Figs. 2(a) to (d), the $\mathrm{A}$-axis is fixed at $A=0^{\circ}$ and the $\mathrm{C}$-axis rotates by $180^{\circ}$ from (i) to (ii). In Figs. $2(\mathrm{e})$ to $(\mathrm{g})$, the A-axis rotates by $-90^{\circ}$ from (i) to (ii). Only in Fig. 2(h), Y-direction displacements are measured for at multiple points with different $\mathrm{X}$ positions to measure the orientation of the test piece's face around the Z-axis.

It is to be emphasized that probing patterns can be modified according to the maximum rotary stroke of rotary axes. The present set of probing patterns assumes that the A-axis rotates for $90^{\circ}$, but not for $180^{\circ}$. As has been discussed in [24], when the A-axis rotates from $-90^{\circ}$ to $90^{\circ}$, all the location errors can be identified by performing fewer probing patterns.

It is also to be noted that the test piece of a square column geometry is merely an example. Many other test pieces, e.g. a cylindrical test piece or a precision sphere, can be used. When such a test piece is used, however, Pattern 4 must be somehow modified, since a sphere (or a circle) does not define the orientation. 


\subsection{Influence of location errors of rotary axes}

The influence of each location error in Table 1 on probed positions will be formulated by showing again Probing pattern 2-a (in Fig. 2(b)) as an example. Location errors are assumed sufficiently small compared to the test piece geometry.

- $\delta x_{A Y}$ : At $A=0^{\circ}, \delta x_{A Y}$ is equivalent to the center offset of C-axis average line to the $\mathrm{X}$-direction from the origin of machine coordinate system. As is illustrated in Fig. 3(a), when $\delta x_{A Y}$ exists (and all the other location errors are zero), the probed position at (ii) becomes $x_{2}^{2 a}=-x_{1}^{2 a}+2 \delta x_{A Y}$. Therefore, we have:

$$
x_{1}^{2 a}+x_{2}^{2 a}=2 \delta x_{A Y}
$$

- $\beta_{A Y}$ : At $A=0^{\circ}, \beta_{A Y}$ is equivalent to the orientation error of the C-axis average line around the $\mathrm{Y}$-axis from the machine coordinate system. Assume that the "nominal" probed position at (i) with $\beta_{A Y}=0$ is given by $\tilde{x}_{1}^{2 a}$. Then, as is illustrated in Fig. $3(\mathrm{~b})$, when $\beta_{A Y}$ exists, the probed position at (i) becomes $x_{1}^{2 a}=\tilde{x}_{1}^{2 a}+\tilde{z}^{2 a} \beta_{A Y}$, where $\tilde{z}^{2 a}$ is the nominal Z-position of the probed point in the machine coordinate system. At (ii), it becomes $x_{2}^{2 a}=-\tilde{x}_{1}^{2 a}+\tilde{z}^{2 a} \beta_{A Y}$. Therefore, we have:

$$
x_{1}^{2 a}+x_{2}^{2 a}=2 \widetilde{z}^{2 a} \beta_{A Y}
$$

The influence of $\beta_{C A}$ is exactly the same as $\beta_{A Y}$ at $A=0^{\circ}$. All the other location errors in Table 1 have no influence at all. To summarize, we have:

$$
x_{1}^{2 a}+x_{2}^{2 a}=2 \delta x_{A Y}+2 \tilde{z}^{2 a}\left(\beta_{A Y}+\beta_{C A}\right)
$$


It is to be noticed that the formulation above is independent on the initial $\mathrm{X}$-position of the test piece, $\tilde{x}_{1}^{2 a}$. The influence of the test piece's location and orientation will be discussed in more details in Section 5 .

All the probing patterns in Fig. 2 can be analogously formulated as follows:

$$
\begin{array}{cc}
\text { Pattern 1 : } & z_{2}^{1}-z_{1}^{1}=2 \tilde{y}^{1} \alpha_{A Y} \\
\text { Pattern 2- a : } & x_{1}^{2 a}+x_{2}^{2 a}=2 \delta x_{A Y}+2 \tilde{z}^{2 a}\left(\beta_{A Y}+\beta_{C A}\right) \\
\text { Pattern 2- b : } & y_{2}^{2 b}+y_{1}^{2 b}=2\left(\delta y_{A Y}+\delta y_{C A}\right)-2 \tilde{z}^{2 b} \alpha_{A Y} \\
\text { Pattern 2- c : } & z_{2}^{2 c}-z_{1}^{2 c}=2 \tilde{x}^{2 c}\left(\beta_{A Y}+\beta_{C A}\right) \\
\text { Pattern 3- a : } & \left.x_{2}^{3 a}-x_{1}^{3 a}=\left(\tilde{z}_{2}^{3 a}-\tilde{z}_{1}^{3 a}\right) \beta_{A Y}+\left(\tilde{y}_{1}^{3 a}-\tilde{y}_{2}^{3 a}\right) \gamma_{A Y}\right) \\
\text { Pattern 3- b : } & z_{2}^{3 b}-y_{1}^{3 b}=\delta z_{A Y}-\delta y_{A Y} \\
\text { Pattern 3- c : } & y_{2}^{3 c}+z_{1}^{3 c}=\delta z_{A Y}+\delta y_{A Y} \\
\text { Pattern 4 : } & \operatorname{angle}_{z}\left(y_{2}^{4}\right)+\operatorname{angle}_{z}\left(y_{1}^{4}\right)=2\left(\gamma_{A Y}+\beta_{C A}\right)
\end{array}
$$

All the symbols are indicated in Figs. 2(a) to (h). The superscript indicates the pattern number (e.g. $z_{1}^{1}$ ). The subscript 1 represents the probed position before the rotation, and the subscript 2 represents the probed position after the rotation (e.g. $z_{1}^{1}$ and $z_{2}^{1}$ ). The symbol ${ }^{\sim}$ represents the nominal position of the target point (e.g. $\tilde{x}_{1}^{2 a}$ ). The function "angle ${ }_{z}$ " in Eq. (13) represents the angle of the least-square mean line of probed positions around the Z-axis.

Eight location errors shown in Table 1 can be uniquely determined by 
solving Eqs. (5) to (13) as follows:

$$
\begin{aligned}
& \delta \hat{x}_{A Y}=\frac{1}{2 \tilde{x}^{2 c}}\left\{\left(x_{1}^{2 a}+x_{2}^{2 a}\right) \tilde{x}^{2 c}-\left(z_{2}^{2 c}-z_{1}^{2 c}\right) \tilde{z}^{2 a}\right\} \\
& \delta \hat{y}_{A Y}=\frac{1}{2}\left\{\left(y_{2}^{3 c}+z_{1}^{3 c}\right)-\left(z_{2}^{3 b}-y_{1}^{3 b}\right)\right\} \\
& \hat{\alpha}_{A Y}=\frac{1}{2 \tilde{y}^{1}}\left(z_{2}^{1}-z_{1}^{1}\right) \\
& \delta \hat{y}_{C A}=\left(y_{2}^{2 b}+y_{1}^{2 b}+2 \tilde{z}^{2 b} \hat{\alpha}_{A Y}\right)-\delta \hat{y}_{A Y} \\
& \delta \hat{z}_{A Y}=\frac{1}{2}\left\{\left(z_{2}^{3 b}-y_{1}^{3 b}\right)+\left(y_{2}^{3 c}+z_{1}^{3 c}\right)\right\} \\
& {\left[\begin{array}{c}
\hat{\beta}_{A Y} \\
\hat{\beta}_{C A} \\
\hat{\gamma}_{A Y}
\end{array}\right]=\left[\begin{array}{ccc}
2 \tilde{x}^{2 c} & 2 \tilde{x}^{2 c} & 0 \\
\tilde{z}_{2}^{3 a}-\tilde{z}_{1}^{3 a} & 0 & \tilde{y}_{1}^{3 a}-\tilde{y}_{2}^{3 a} \\
0 & 2 & 2
\end{array}\right]^{-1}\left[\begin{array}{c}
z_{2}^{2 c}-z_{1}^{2 c} \\
x_{2}^{3 a}-x_{1}^{3 a} \\
\operatorname{angle}_{z}\left(y_{2}^{4}\right)+\operatorname{angle}_{z}\left(y_{1}^{4}\right)
\end{array}\right]}
\end{aligned}
$$

\section{Experimental case study}

\subsection{Experimental setup}

The present error calibration scheme is applied to a commercial middlesize 5-axis machining center of the configuration shown in Fig. 1. Table 2 shows its major specifications. A touch-trigger probe, RMP-60 by Renishaw plc. [25], is used in experiments. RMP-60 is a typical touch-trigger probe for machining centers, detecting the contact by the spring-loaded kinematic arrangement of rods and balls. A ruby sphere of the diameter $6 \mathrm{~mm}$ is used as a probe ball. Table 3 shows major specifications of the probe. Figure 4 shows the experimental setup. An aluminum alloy (JIS A5052) block of the size about $100 \times 100 \times 150 \mathrm{~mm}$ is used as the test piece. 


\subsection{Measurement procedure}

To reduce the influence of the machine tool's or the probe's repeatability error or geometric/surface inaccuracy of the test piece, it is favorable to probe multiple points and average them. For this reason, all the points shown in Fig. 5 are probed.

The test procedure is described as follows:

1. The test piece is fixed on the machine table. The center of the test piece's upper surface is located approximately at $(\mathrm{X}, \mathrm{Y}, \mathrm{Z})=(0,-200,170)$ $\mathrm{mm}$ in the machine coordinate system. The machine coordinate system is defined in Section 3.1. Align the test piece to the machine's linear axes only roughly.

2. At (I) $A_{i}=0^{\circ}$ and $C_{j}=0^{\circ}$, total 28 points ( 12 points on the top face, and four points on each side face) are probed from the direction normal to the surface, as illustrated in Fig. 5 (I) and Fig. 6.

3. Then, index the C-axis at (II) $A_{i}=0^{\circ}, C_{j}=90^{\circ}$ and probe same 28 points as illustrated in Fig. 5 (II). Figure 6 shows the nominal position of probed points, as well as the probing sequence, in the workpiece coordinate system. The same probing sequence is applied to all $(\mathrm{I}) \sim(\mathrm{VII})$ in the workpiece coordinate system. Perform the same probing sequence at (III) $A_{i}=0^{\circ}, C_{j}=180^{\circ}$, and (IV) $A_{i}=0^{\circ}, C_{j}=270^{\circ}$.

4. For (V) $A_{i}=-90^{\circ}, C_{j}=90^{\circ}$, (VI) $A_{i}=-90^{\circ}, C_{j}=180^{\circ}$, and (VII) $A_{i}=-90^{\circ}, C_{j}=270^{\circ}, 14$ points are probed at each indexed angle, as depicted in Fig. 5 (V)-(VII), to avoid unwanted interference of the probe to the test piece. 
Total 154 points are probed. The total measurement time was about 20 minutes. In the experiment, this set of measurement was repeated four times to observe the repeatability of the measurement.

For $A_{i}=0^{\circ},-90^{\circ}(i=1,2)$, and $C_{j}=0^{\circ}, 90^{\circ}, 180^{\circ}, 270^{\circ}(j=1,2,3,4)$, the probed position is denoted by:

$$
p(i, j, k)=[x(i, j, k) \quad y(i, j, k) \quad z(i, j, k)]^{T}
$$

where the index $k$ labels probed points as shown in Fig. $6(k=1 \sim 28)$. Since the probing is one-dimensional, only one of $x(i, j, k), y(i, j, k)$, and $z(i, j, k)$ is measured, and the others represent the nominal position.

\subsection{Measurement result}

Figures 7 (a) and (b) show probed positions for (I) $C_{j}=0^{\circ}$, (II) $90^{\circ}$, (III) $180^{\circ}$, and (IV) $270^{\circ}$ with $A_{i}=0^{\circ}$. Nominal target positions are represented by black dots ".". Their measured positions are shown by "o". The error from nominal to measured positions is magnified 25 times. Figs. 7(a) and (b) respectively show the projection to the XY plane and the YZ plane.

For each face of the test piece, the least-square average surface of measured positions is calculated, which is represented by the polyhedron in green shown in Figs. 7(a) and (b). Since side faces of the test piece are measured at only four points in the same Z-height, their inclination around X- or Y-axes cannot be seen. In Fig. 7, all side faces of the polyhedra are assumed to be vertical.

For example, at (I) in Fig. 7 (a), the center of the test piece is shifted by about $0.5 \mathrm{~mm}$ in both $\mathrm{X}$ - and $\mathrm{Y}$-directions. This represents the setup error 
in the test piece position from its nominal position. Furthermore, at (I) in Fig. 7(b), it is clearly observed that the top surface of the test piece is tilted by about $0.2 \mathrm{~mm} / 100 \mathrm{~mm}$ around the $\mathrm{Y}$-axis. This indicates the geometric inaccuracy of the test piece.

Such setup or geometric errors of test piece itself do not affect the identification of location errors in principle. To remove their influence, the displacement at each probed point in relative to that at the corresponding point in (I) $C_{1}=0^{\circ}$ and $A_{1}=0^{\circ}$ is calculated by:

$$
\Delta p(i, j, k)=p(i, j, k)-D_{a}\left(-A_{i}\right) D_{c}\left(-C_{j}\right) p(1,1, k)
$$

Equations (6) to (13) are constructed by averaging corresponding points for each pattern. For example, $x_{1}^{2 a}$ and $x_{2}^{2 a}$ for Pattern 2-a (Fig. 2(b)) are given as the mean of corresponding probed coordinates in (IV) and (II) in Fig. 5, i.e. $x_{1}^{2 a}=\operatorname{mean}_{k=1 \sim 4,9 \sim 12} \Delta x(4,1, k)$, and $x_{2}^{2 a}=\operatorname{mean}_{k=1 \sim 4,9 \sim 12} \Delta x(2,1, k)$.

Figures $8(\mathrm{a})$ to $(\mathrm{d})$ show probed relative displacements, $\Delta p(i, j, k)$, calculated from Fig. 7. Figures 8(a), (b), and (c) respectively show the projection onto XY, XZ, and YZ planes, and (d) shows its three-dimensional view. The error from nominal to measured positions is magnified by 500 times.

Similarly, Figs. 9(a) and (b) shows measured results at $A=-90^{\circ}$, i.e. (V) $A_{i}=-90^{\circ}, C_{j}=90^{\circ},(\mathrm{VI}) A_{i}=-90^{\circ}, C_{j}=180^{\circ}$, and (VII) $A_{i}=-90^{\circ}$, $C_{j}=270^{\circ}$. Figures $9(\mathrm{a})$ shows the projection onto the XY plane, and (b) shows its three-dimensional view. 


\subsection{Observation and identification of location errors}

From Figs. 8 and 9, many intuitive observations can be made on error motions of rotary axes. For example, in Fig. 8(a), the shift in X- and Y-directions observed in (II), (III), and (IV) clearly indicates the miscalibration of the $\mathrm{C}$-axis center of rotation, i.e. $\delta x_{A Y}\left(\delta x_{C A}\right)$ and $\delta y_{A Y}$. In Fig. $8(\mathrm{c})$, the inclination of the top surface around the $\mathrm{X}$-axis is observed in (II)(III)(IV), which suggests the squareness error of C-axis to Y-axis, i.e. $\alpha_{A Y}$. In Fig. 9(a), the test piece is shifted in + Y-direction by about $40 \mu \mathrm{m}$, which suggests the miscalibration of the A-axis center, i.e. $\delta z_{A Y}$ and $\delta y_{A Y}$.

From Eqs. (6) to (13), location errors of rotary axes are identified. To check the repeatability of the measurement, total four sets of same experiments were repeated, and location errors identified by each set are shown in Table 4. The mean of identified location errors and their maximum variation from the mean are also shown. The identified values of $\delta z_{A Y}$ show relatively large variation, which may be caused by the thermal deformation of the machine in the Z-direction. All other parameters have the variation at most $\pm 2.1 \mu \mathrm{m}$ (for linear errors) or $\pm 0.5 \mathrm{mdeg}$ (for angular errors). The uncertainty analysis will be presented in Section 5 .

\subsection{Validation of identified location errors}

Among location errors identified in Table $4, \delta x_{A Y}, \delta y_{A Y}$, and $\delta z_{A Y}$ can be modified by simply shifting the origin of the machine coordinate system. $\alpha_{A Y}$ can be modified by offsetting the A-axis angular command.

To validate the accuracy of these four estimates, the identified $\delta x_{A Y}, \delta y_{A Y}$, $\delta z_{A Y}$, and $\alpha_{A Y}$ at the fourth test are compensated as described above. Then, 
the same probing sequence was repeated to re-identify location errors, as also shown in Table 4 ("Identified value after compensating $\delta x_{A Y}, \delta y_{A Y}, \delta z_{A Y}$, $\left.\alpha_{A Y}{ }^{\prime \prime}\right)$. The values with $*$ correspond to compensated parameters. Compared to identified values before the compensation, they are significantly reduced. Assuming that the positioning error of linear axes for shifting the machine coordinate system is sufficiently small, it suggests the validity of the identified values. The residues are at most $2.4 \mu \mathrm{m}$, which is within the repeatability error of the machine's positioning or the probing.

\section{Uncertainty analysis}

The probing patterns presented in Fig. 2 nominally probe same points on the test piece at each $A_{i}$ and $C_{j}$. The calibration of location errors presented in Section 3.2 is based on the measured displacement of each point from its initial position (measured at $A_{i}=C_{j}=0^{\circ}$ ) in the workpiece coordinate system. Therefore, the geometric inaccuracy of the test piece or its setup (alignment) error does not impose significant effect on the estimates of location errors. This is an important feature of the proposed approach. On the other hands, as was stated in Section 2.2, the present scheme assumes negligibly small geometric errors of linear axes. Error motions of linear axes may have significant influence on the estimates.

To validate it, this section presents the uncertainty analysis of identified location errors. The principal contributors to the uncertainty are listed in Table 5. Associated with the probing, only the unidirectional repeatability (random measurement error) is taken into account; the measurement error 
caused by the directional pre-travel variation or other factors are neglected assuming proper pre-calibration and compensation [18]. Note that the influence of the misalignment of the probe sphere from the spindle average line (i.e. run-out), or constant tilting of the probe stylus, is assumed to be negligibly small by proper alignment.

The main focus of this analysis is to assess the influence of error motions of linear axes. Error motions of linear axes are modelled as follows, analogously as in [26]:

$$
\begin{aligned}
& E_{x x}(x)=E_{x x, l i n} \cdot x+\operatorname{interpolate}\left(X_{i}, E_{x x, \text { rand }}\left(X_{i}\right), x\right) \\
& E_{y x}(x)=\operatorname{interpolate}\left(X_{i}, E_{y x, \text { rand }}\left(X_{i}\right), x\right) \\
& E_{z x}(x)=\operatorname{interpolate}\left(X_{i}, E_{z x, \text { rand }}\left(X_{i}\right), x\right)
\end{aligned}
$$

where $E_{x x}(x), E_{y x}(x)$, and $E_{z x}(x)$ respectively represent positioning errors in $\mathrm{X}, \mathrm{Y}$, and $\mathrm{Z}$ directions of $\mathrm{X}$-axis at the nominal position, $x$. The function interpolate represents the linear interpolation of the function $E_{*}\left(X_{i}\right)$ at $x$. $X_{i}$ is given by $X_{i}=p_{\text {rand }} \cdot i$ where $p_{\text {rand }}$ is a random number uniformly distributed in $[20,50] \mathrm{mm}$. The squareness errors of linear axes are also taken into account. For example, the positioning error of Y-axis to the X-direction is modelled by:

$$
E_{x y}(y)=S_{x y} \cdot y+\operatorname{interpolate}\left(Y_{i}, E_{x y, r a n d}\left(Y_{i}\right), y\right)
$$

Error motions of all the linear axes are modelled analogously. Angular errors of linear axes are neglected, assuming small-sized machines. The magnitude of each error is chosen randomly for each run with the normal distribution given in Table 5. The error ranges are taken from comparative measurements, manufacturer specifications or from values stated in standards. With 
this model of linear axes, the whole calibration chain is simulated. At each probing point, the machine's positioning error and the test piece's position and orientation are calculated, and location errors are identified by exactly the same procedure presented in Sections 4.2 and 4.3. Monte Carlo simulations are used [26] with 1,000 runs to assess the uncertainty in the estimates.

Table 5 shows the quantitative influence of each contributor to the uncertainty in the estimate of $\delta \hat{y}_{A Y}$ and $\hat{\beta}_{A Y}$ (only two location errors are shown as examples). For example, $\delta \hat{y}_{A Y}$ is estimated from Patterns 3-b and 3-c (Eq.(15)), From Figs. 2(f) and 2(g). it can be easily understood that the squareness of Z- and Y-axes has significantly larger influence. Position and orientation errors of test piece setup have only negligibly small influence.

Table 6 shows the uncertainty in all the estimated location errors. It is to be noted that $\hat{\beta}_{A Y}, \hat{\gamma}_{A Y}$, and $\hat{\beta}_{C A}$ have larger uncertainty mainly because their estimation requires Pattern 4 (see Eq. (19)). Pattern 4 (Fig. 2(h)) requires the angular measurement, whose uncertainty gets larger as $\tilde{L}_{1}^{4}$ becomes smaller.

\section{Conclusion}

This paper proposes a scheme to calibrate location errors of rotary axes by on-the-machine measurement of the test piece by using a contact-type touch-trigger probe installed on the machine's spindle. Similarly as many other methodologies reported in the literature on the calibration of rotary axes (e.g. ball bar measurement and the R-test), the present scheme measures the relative displacement of the spindle to the table, and it is therefore 
not possible in principle to separate error motions of rotary axes and linear axes. The uncertainty analysis was presented to quantitatively assess the influence of error motions of linear axes on the estimates of location errors.

Compared to conventional calibration schemes described in ISO/DIS 107911:2012 and ISO/DIS 10791-6:2012, where a precision sphere and a linear displacement sensor or the ball bar are used, the proposed approach is more suitable to efficient and automated calibration procedure of location errors. It is therefore advantageous in the application to periodic check of location errors, or periodic updating of their numerical compensation.

As was discussed in Section 5, the geometric inaccuracy and the setup error of the test piece impose only negligibly small influence on the estimates. Therefore, there is no need to use a calibrated artefact to perform the present scheme. For example, a part of the workpiece to be machined, the fixture, or the machine table can be the target of probing as long as its surface quality is reasonably high. Such an application should be further studied in our future research to facilitate its application to periodic check by machine tool users.

Location errors only represent mean location and orientation of axis of rotation. The location and the orientation may vary with its rotation (described by the term "axis of rotation error motion" in ISO 230-7 [3]). The "error map" of rotary axes can be constructed by extending the present calibration scheme. It will be studied in our future research. 


\section{References}

[1] ISO 10791-1:1998, Test conditions for machining centres - Part 1: Geometric tests for machines with horizontal spindle and with accessory heads (horizontal Z-axis), 1998.

[2] ISO/DIS 10791-1:2012, Test conditions for machining centres - Part 1: Geometric tests for machines with horizontal spindle (horizontal Z-axis), 2012.

[3] ISO 230-7:2006, Test code for machine tools - Part 7: Geometric accuracy of axes of rotation, 2006.

[4] Y. Abbaszaheh-Mir, J. R. R. Mayer, G. Clotier, C. Fortin, Theory and simulation for the identification of the link geometric errors for a fiveaxis machine tool using a telescoping magnetic ball-bar, International Journal of Production Research 40 (18) (2002) 4781-4797.

[5] S. H. H. Zargarbashi, J. R. R. Mayer, Single setup estimation of a five axis machine tool eight link errors by programmed end point constraint and on the fly measurement with Capball sensors, International Journal of Machine Tools and Manufacture 49 (2009) 759-766.

[6] M. S. Uddin, S. Ibaraki, A. Matsubara, T. Matsushita, Prediction and compensation of machining geometric errors of five-axis machining centers with kinematic errors, Precision Engineering 33 (2) (2009) 194-201.

[7] S. C. Veldhuis, M. A. Elbestawi, A strategy for the compensations of the errors in five-axis machining, Annals of the CIRP 44 (1) (1995) 373-377. 
[8] R. M. D. Mahbubur, J. Heikkala, K. Lappalainen, J. A.Karjalainen, Positioning accuracy improvement in five-axis milling by postprocessing, International Journal of Machine Tools and Manufacture 37 (2) (1997) $223-236$.

[9] S. Ibaraki and W.Knapp, Indirect Measurement of Volumetric Accuracy for Three-axis and Five-axis Machine Tools: A Review, Int'l J. of Automation Technology 6 (2) (2012).

[10] H. Schwenke, W. Knapp, H. Haitjema, A. Weckenmann, R. Schmitt, F. Delbressine, Geometric error measurement and compensation of machines -An update, CIRP Annals - Manufacturing Technology 57 (2) (2008) 560-575.

[11] Y. Kakino, T. Ihara, H. Sato, H. Otsubo, A Study on the motion accuracy of NC machine tools (7th report) -Measurement of motion accuracy of 5-axis machine by DBB tests-, Journal of Japan Society for Precision Engineering 60 (5) (1994) 718-723 (in Japanese).

[12] M. Tsutsumi, A. Saito, Identification and compensation of systematic deviations particular to 5-axis machining centers, International Journal of Machine Tools and Manufacture 43 (2003) 771-780.

[13] W. T. Lei, M. P. Sung, W. L. Liu, Y. C. Chuang, Double ballbar test for the rotary axes of five-axis CNC machine tools, International Journal of Machine Tools and Manufacture 47 (2007) 273-285.

[14] ISO/DIS 10791-6:2012 Test conditions for machining centres - Part 6: Accuracy of feeds, speeds and interpolations, 2012. 
[15] S. Weikert, R-Test, a New Device for Accuracy Measurements on Five Axis Machine Tools, Annals of the CIRP 53 (1) (2004) 429-432.

[16] B. Bringmann, W. Knapp, Model-based Chase-the-Ball' calibration of a 5-axis machining center, Annals of the CIRP, 55 (1) (2006) 531-534.

[17] S. Ibaraki, C. Oyama, H. Otsubo, Construction of an error map of rotary axes on a five-axis machining center by static R-test, International Journal of Machine Tools and Manufacture, 51 (2011) 190-200.

[18] ISO/DIS 230-10:2009, Test code for machine tools - Part 10: Determination of measuring performance of probing systems of numerically controlled machine tools, 2009.

[19] G. X. Zhang, Y. F. Zang, Method for machine geometry calibration using 1-D ball array. CIRP Annals - Manufacturing Technology 40(1) (1991) 519-522.

[20] J. B. De Aquino Silva, M. Burdekin, A modular space frame for assessing the performance of co-ordinate measuring machines (CMMs), Precision Engineering 26 (1) (2002), 37-48.

[21] B. Bringmann A. Kung, A measuring artefact for true 3D machine testing and calibration, CIRP Annals - Manufacturing Technology 54 (1) (2005) 471-474.

[22] T. Erkan, J. R. R. Mayer, Y. Dupont, Volumetric distortion assessment of a five-axis machine by probing a 3D reconfigurable uncalibrated master ball artefact, Precision Engineering, 35 (2011) 116-125. 
[23] I. Inasaki, K. Kishinami, S. Sakamoto, N. Sugimura, Y. Takeuchi, F. Tanaka, Shaper generation theory of machine tools - its basis and applications, Yokendo, Tokyo, 1997 (in Japanese).

[24] S. Ibaraki, M. Sawada, A. Matsubara, T. Matsushita, Machining tests to identify kinematic errors on five-axis machine tools, Precision Engineering 34 (3) (2010), 387-398.

[25] RMP-60 - radio machine probe (data sheet H-2000-2122-04-A), Renishaw, plc., 2008.

[26] B. Bringmann, W. Knapp, Machine tool calibration: Geometric test uncertainty depends on machine tool performance, Precision Engineering, 33 (2009) 524-529. 


\section{List of Tables}

1 Location errors for the machine configuration in Fig. 1. . . . . 27

2 Machine tool specifications. . . . . . . . . . . . 28

3 Touch-trigger probe specifications (RMP-60 by Renishaw [25]). 29

4 Identified location errors of rotary axes. . . . . . . . . . . 30

$5 \quad$ Contributors for identification uncertainties. . . . . . . . . . 31

6 Combined uncertainty $(k=1)$ in the estimation of each loca-

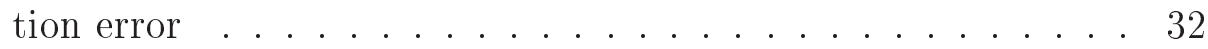

\section{List of Figures}

1 The configuration of the five-axis machine tool considered in this

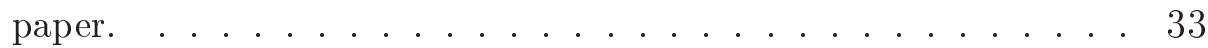

2 Probing patterns. (a) Pattern 1, (b) Pattern 2-a, (c) Pattern 2-b, (d) Pattern 2-c, (e) Pattern 3-a, (f) Pattern 3-b, (g) Pattern 3-c,

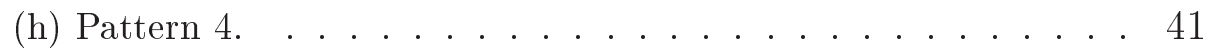

3 The influence of $\mathrm{C}$-axis location errors on probed positions. (a) Influence of $\delta x_{A Y}$, (b) Influence of $\beta_{A Y} \ldots \ldots$. . . . . . . . 42

4 Experimental setup. . . . . . . . . . . . . . . 43

5 Probed points at each index angle of $\mathrm{A}$ and $\mathrm{C}$ axes. . . . . . . . . 44

6 Position of probed points and probing sequence in the workpiece coordinate system. . . . . . . . . . . . . 45

7 "Raw" probed positions for (I) to (IV) $\left(A=0^{\circ}, C=0,90,180,270^{\circ}\right)$. Errors are magnified 25 times. (a) Projection onto XY plane. (b) Projection onto XZ plane. 
8 Probed positions for (II) to (IV) $\left(A=0^{\circ}, C=90,180,270^{\circ}\right)$ in relative to corresponding points in (I) $\left(C=0^{\circ}\right)$. Errors are magnified 500 times. (a) Projection onto XY plane. (b) Projection onto XZ plane, (c) Projection onto YZ plane, (d) In three-dimensional view (only average planes of probed points are shown). . . . . . 48

9 Probed positions for (V) to (VII) $\left(A=-90^{\circ}, C=90,180,270^{\circ}\right)$ in relative to corresponding points in (I) $\left(A=0^{\circ}, C=0^{\circ}\right)$. Errors are magnified 500 times. (a) Projection onto XY plane. (b) In three-dimensional view (only average planes of probed points are

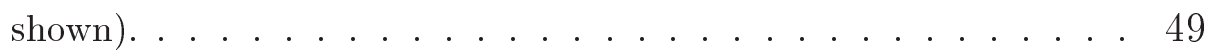


Table 1: Location errors for the machine configuration in Fig. 1.

\begin{tabular}{l|l|l}
\hline Symbol [23] & Symbol [3] & Description \\
\hline \multicolumn{2}{l}{ Location errors associated with rotary axes } \\
\hline$\alpha_{A Y}$ & A0A & Angular positioning error of A-axis at $A=0^{\circ}$ \\
$\beta_{A Y}$ & B0A & Squareness error of A- to Z-axis \\
$\gamma_{A Y}$ & C0A & Squareness error of A- to Y-axis \\
$\beta_{C A}$ & B0C-B0A & Squareness error of C- to A-axis \\
$\delta x_{A Y}$ & X0A & Linear offset of A-axis in X direction \\
$\delta y_{A Y}$ & Y0A & Linear offset of A-axis in Y direction \\
$\delta z_{A Y}$ & Z0A & Linear offset of A-axis in Z direction \\
$\delta y_{C A}$ & Y0C-Y0A & Linear offset of C-axis from A-axis in Y \\
\hline
\end{tabular}


Table 2: Machine tool specifications.

\begin{tabular}{l|l}
\hline Stroke & $\begin{array}{l}\mathrm{X}: 1250 \mathrm{~mm}, \mathrm{Y}: 660 \mathrm{~mm}, \mathrm{Z}: 540 \mathrm{~mm}, \\
\mathrm{~A}:+20^{\circ} \sim-110^{\circ}, \mathrm{C}: 360^{\circ} .\end{array}$ \\
\hline Table size & $\phi 500 \mathrm{~mm}$ \\
\hline Drives & $\begin{array}{l}\mathrm{X}, \mathrm{Y}, \text { and Z: rotary servo motor with ballscrew, } \\
\text { A and } \mathrm{C}: \text { direct drive }\end{array}$ \\
\hline
\end{tabular}


Table 3: Touch-trigger probe specifications (RMP-60 by Renishaw [25]).

\begin{tabular}{l|l}
\hline Primary application & inspection probe for machining centers \\
\hline Sense directions & Omni-directional $\pm \mathrm{X}, \pm \mathrm{Y}, \pm \mathrm{Z}$. \\
\hline $\begin{array}{l}\text { Repeatability (in catalog; } \\
\text { max } 2 \sigma \text { value in any direction) }\end{array}$ & $1.0 \mu \mathrm{m}$ \\
\hline Stylus length & $50 \mathrm{~mm}$ \\
\hline Signal transmission & $\begin{array}{l}\text { Frequency hopping spread } \\
\text { spectrum (FHSS) radio }\end{array}$ \\
\hline
\end{tabular}


Table 4: Identified location errors of rotary axes.

\begin{tabular}{|c|c|c|c|c|c|c|c|}
\hline \multirow[t]{2}{*}{ Location error } & \multicolumn{4}{|c|}{$\begin{array}{c}\text { Identified value } \\
\text { at each test }\end{array}$} & \multirow{2}{*}{$\begin{array}{l}\text { Identified } \\
\text { value } \\
\text { (mean) } \\
\end{array}$} & \multirow{2}{*}{$\begin{array}{l}\text { Max. and min. } \\
\text { deviation from } \\
\text { mean } \\
\end{array}$} & \multirow{2}{*}{$\begin{array}{c}\text { Identified value } \\
\text { after compensating } \delta x_{A Y}, \\
\delta y_{A Y}, \delta z_{A Y}, \alpha_{A Y}(*)\end{array}$} \\
\hline & $1 \mathrm{st}$ & 2 nd & $3 \mathrm{rd}$ & 4 th & & & \\
\hline$\delta x_{A Y}(\mu \mathrm{m})$ & 15.0 & 15.0 & 15.7 & 12.5 & 14.6 & \pm 2.1 & $2.0 *$ \\
\hline$\delta y_{A Y}(\mu \mathrm{m})$ & 24.1 & 22.5 & 24.0 & 22.1 & 23.2 & \pm 1.0 & $-2.4 *$ \\
\hline$\delta z_{A Y}(\mu \mathrm{m})$ & 22.1 & 24.4 & 27.2 & 37.1 & 27.7 & \pm 9.4 & $-0.2 *$ \\
\hline$\delta y_{C A}(\mu \mathrm{m})$ & 3.9 & 4.2 & 4.7 & 2.6 & 3.9 & \pm 1.2 & 4.4 \\
\hline$\alpha_{A Y}(\mathrm{mdeg})$ & -0.6 & -0.7 & -0.3 & -0.6 & -0.5 & \pm 0.3 & $0.0 *$ \\
\hline$\beta_{A Y}$ (mdeg) & -0.1 & -0.1 & -0.3 & 0.2 & -0.1 & \pm 0.3 & -0.1 \\
\hline$\gamma_{A Y}$ (mdeg) & -0.6 & -0.7 & -0.6 & -0.1 & -0.5 & \pm 0.4 & -0.5 \\
\hline$\beta_{C A}$ (mdeg) & 0.4 & 0.3 & 0.5 & -0.3 & 0.2 & \pm 0.5 & 0.3 \\
\hline
\end{tabular}


Table 5: Contributors for identification uncertainties.

\begin{tabular}{|c|c|c|c|}
\hline \multirow[t]{2}{*}{ Contributors } & \multirow{2}{*}{$\begin{array}{l}\text { Standard } \\
\text { uncertainty }\end{array}$} & \multicolumn{2}{|c|}{ Contribution to uncertainty } \\
\hline & & $\hat{\delta} y_{A Y}(\mu \mathrm{m})$ & $\hat{\beta}_{A Y}(\mathrm{mdeg})$ \\
\hline \multicolumn{4}{|l|}{ Probing } \\
\hline - unidirectional repeatability & $0.7 \mu \mathrm{m}$ & 0.3 & 0.5 \\
\hline - directional pre-travel variation & 0 & 0 & 0 \\
\hline \multicolumn{4}{|l|}{ Linear axes, $\mathrm{X}$-axis } \\
\hline - linear error, $E_{x x, l i n}$ & $1 \mu \mathrm{m} / \mathrm{mm}$ & 0 & 0 \\
\hline - non-systematic error, $E_{x x, r a n d}$ & $2 \mu \mathrm{m}$ & 0 & 0 \\
\hline - straightness error, $E_{y x, \text { rand }}, E_{z x, \text { rand }}$ & $2 \mu \mathrm{m}$ & $1.6,0.2$ & $0.0,0.3$ \\
\hline Y-axis & & & \\
\hline - linear error, $E_{y y, l i n}$ & $1 \mu \mathrm{m} / \mathrm{mm}$ & 0.4 & 0.0 \\
\hline - non-systematic error, $E_{y y, \text { rand }}$ & $2 \mu \mathrm{m}$ & 0.6 & 0.0 \\
\hline - straightness error, $E_{x y, r a n d}, E_{z y, r a n d}$ & $2 \mu \mathrm{m}$ & $0,1.7$ & $0.4,0$ \\
\hline - squareness of Y- to X-axis, $S_{x y}$ & $3 \mu \mathrm{m} / 100 \mathrm{~mm}$ & 0 & 1.5 \\
\hline \multicolumn{4}{|l|}{ Z-axis } \\
\hline - linear error, $E_{z z, l i n}$ & $1 \mu \mathrm{m} / \mathrm{mm}$ & 0.4 & 0 \\
\hline - non-systematic error, $E_{z z, \text { rand }}$ & $2 \mu \mathrm{m}$ & 0.6 & 0 \\
\hline - straightness error, $E_{x z, \text { rand }}, E_{y z, \text { rand }}$ & $2 \mu \mathrm{m}$ & $0,1.5$ & $0.3,0.0$ \\
\hline - squareness of Z- to X-axis, $S_{x z}$ & $3 \mu \mathrm{m} / 100 \mathrm{~mm}$ & 0 & 0.2 \\
\hline - squareness of Z- to Y-axis, $S_{y z}$ & $3 \mu \mathrm{m} / 100 \mathrm{~mm}$ & 5.5 & 0 \\
\hline \multicolumn{4}{|l|}{ Test piece } \\
\hline - position error in $\mathrm{X}, \mathrm{Y}, \mathrm{Z}$ & $10,10,120 \mu \mathrm{m}$ & $0,0,0$ & $0,0,0.0$ \\
\hline - orientation error around X, Y, Z & $10,10,10 \mu \mathrm{m} / 100 \mathrm{~mm}$ & $0,0,0$ & $0.0,0.0,0$ \\
\hline - influence of surface roughness to probing & $1 \mu \mathrm{m}$ & 0.5 & 0.7 \\
\hline
\end{tabular}


Table 6: Combined uncertainty $(k=1)$ in the estimation of each location error

\begin{tabular}{l|l|l|l|l}
\hline & \multicolumn{4}{|c}{ Location errors } \\
\hline \hline & $\hat{\delta} x_{A Y}$ & $\hat{\delta} y_{A Y}$ & $\hat{\delta} z_{A Y}$ & $\hat{\delta} y_{C A}$ \\
\hline Uncertainty & $5.7 \mu \mathrm{m}$ & $6.0 \mu \mathrm{m}$ & $4.6 \mu \mathrm{m}$ & $3.5 \mu \mathrm{m}$ \\
\hline \hline & $\hat{\alpha}_{A Y}$ & $\hat{\beta}_{A Y}$ & $\hat{\gamma}_{A Y}$ & $\hat{\beta}_{C A}$ \\
\hline Uncertainty & $0.3 \mathrm{mdeg}$ & $2.0 \mathrm{mdeg}$ & $1.6 \mathrm{mdeg}$ & $1.9 \mathrm{mdeg}$ \\
\hline
\end{tabular}




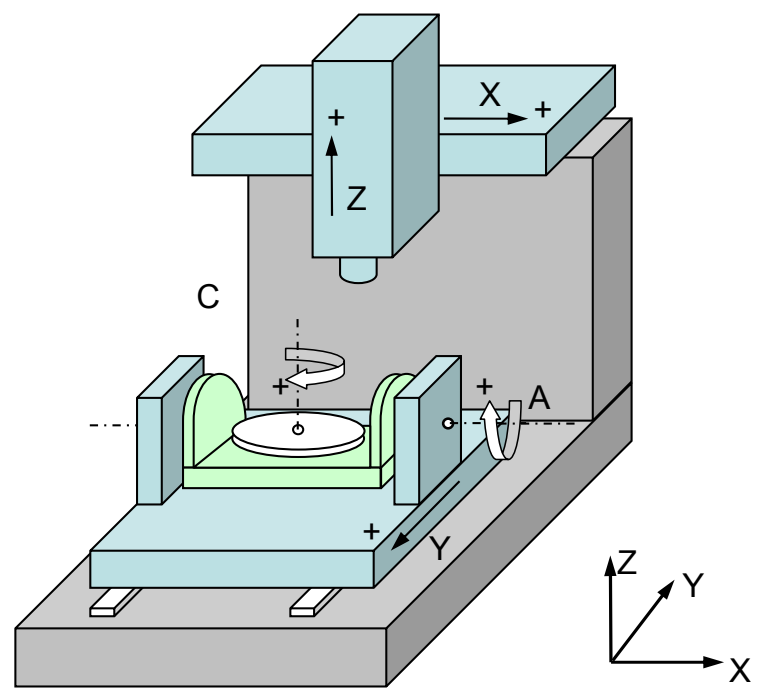

Figure 1: The configuration of the five-axis machine tool considered in this paper. 


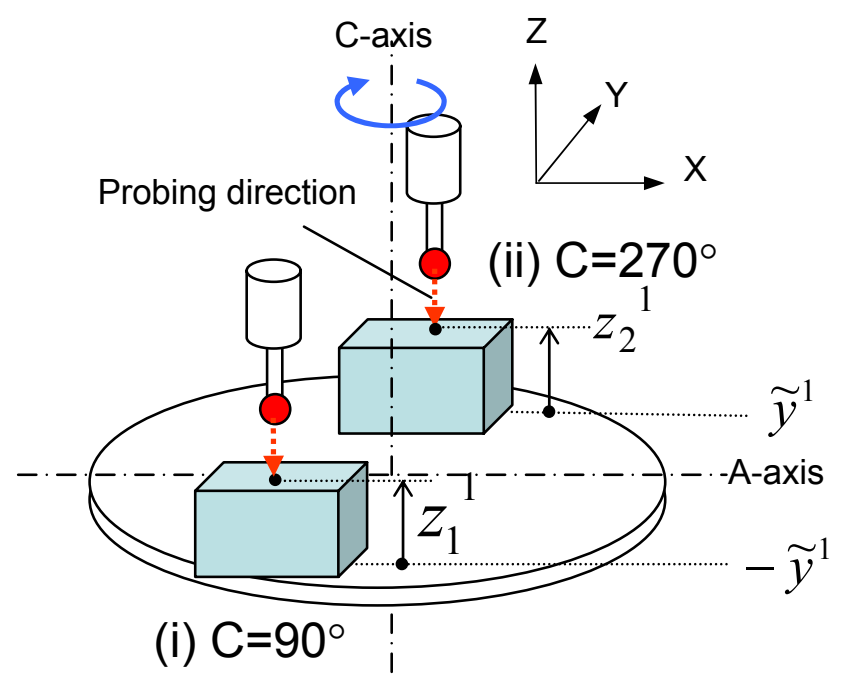

(a) Pattern 1. 


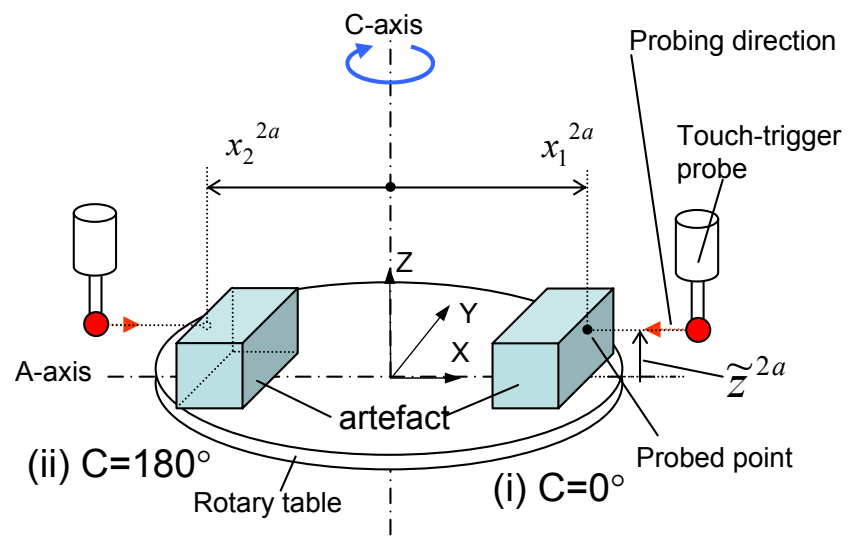

(b) Pattern 2-a. 


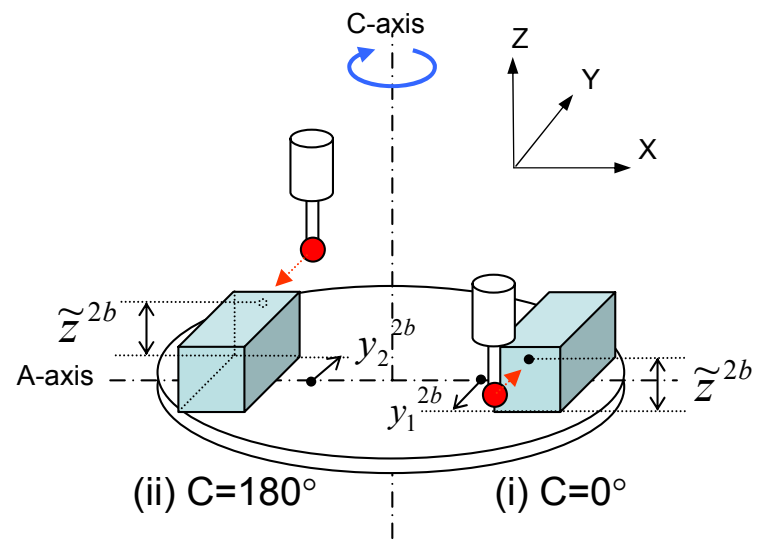

(c) Pattern 2-b. 


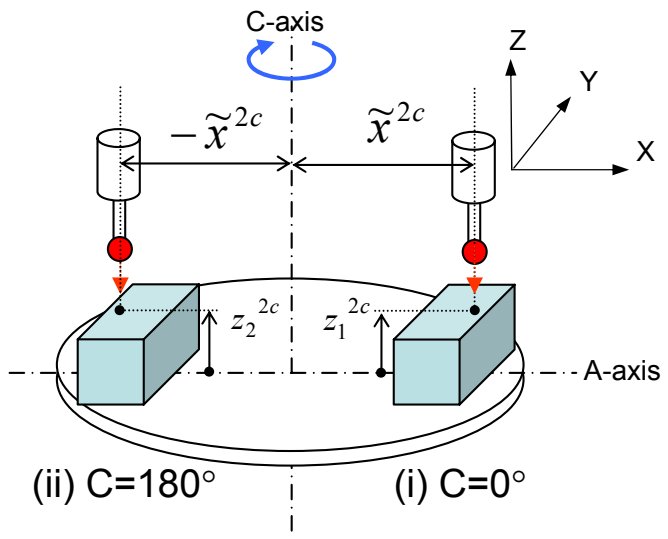

(d) Pattern 2-c. 


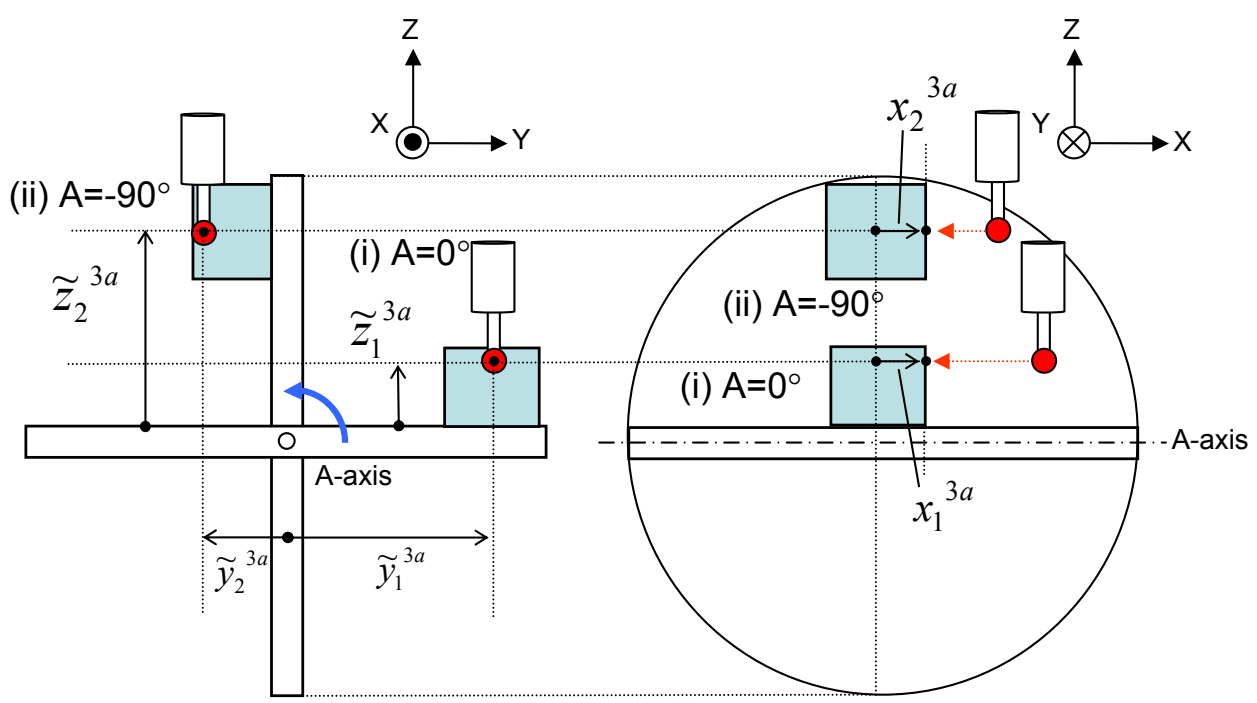

(e) Pattern 3-a. 


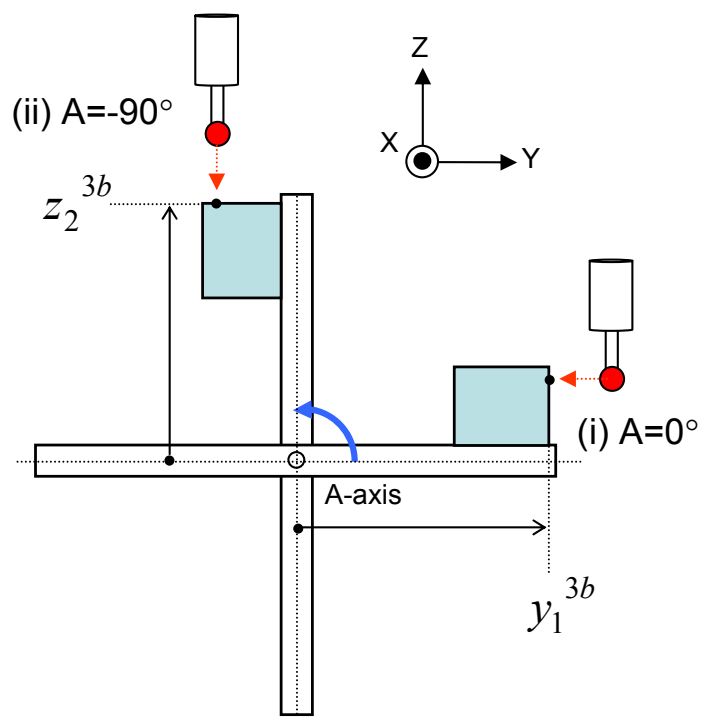

(f) Pattern 3-b. 


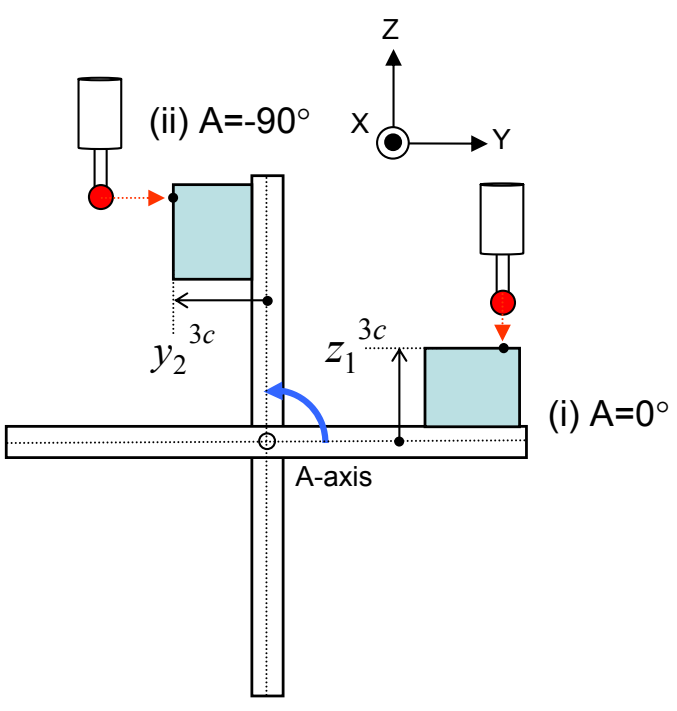

(g) Pattern 3-c. 


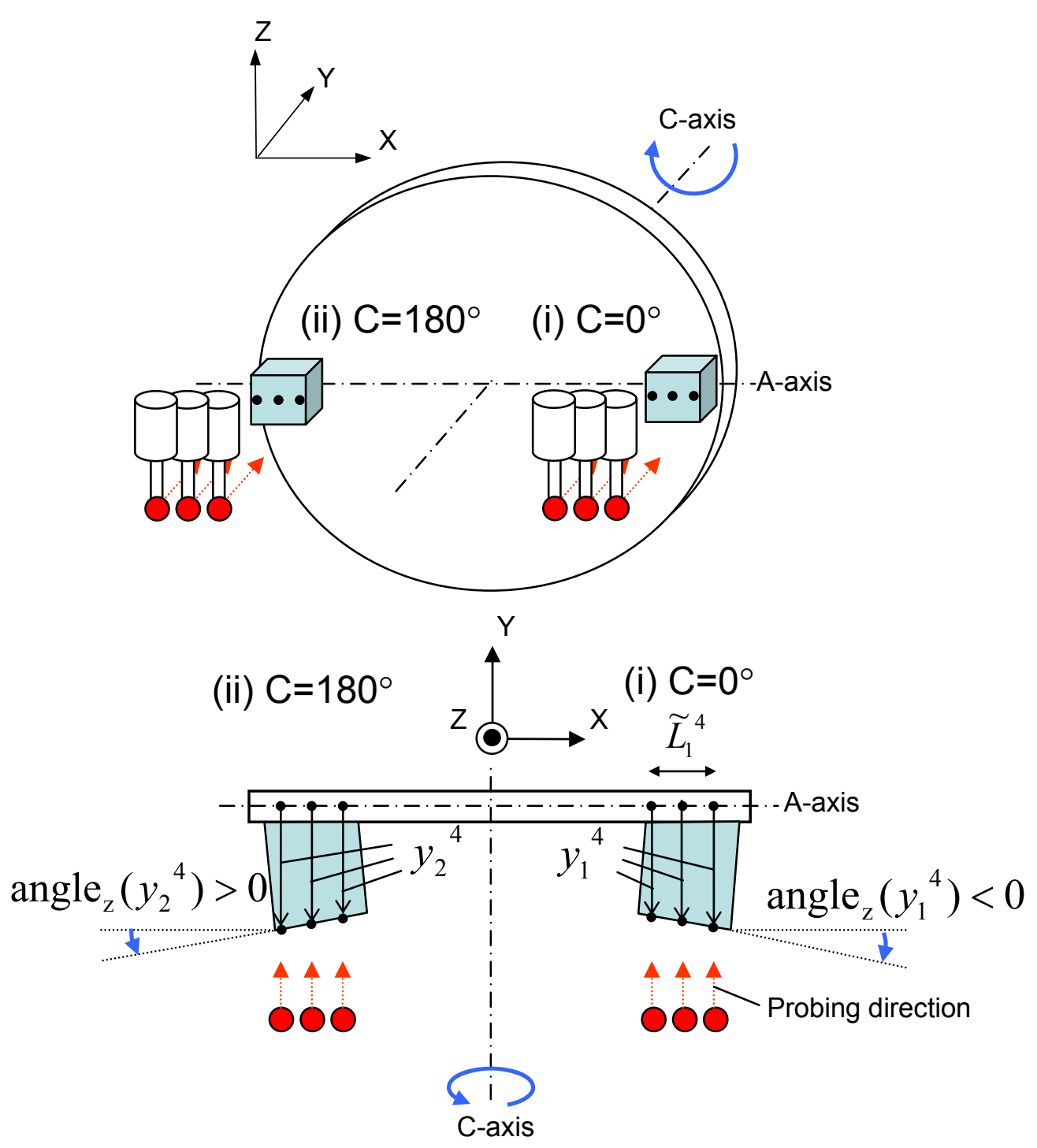

(h) Pattern 4.

Figure 2: Probing patterns. (a) Pattern 1, (b) Pattern 2-a, (c) Pattern 2-b, (d) Pattern 2-c, (e) Pattern 3-a, (f) Pattern 3-b, (g) Pattern 3-c, (h) Pattern 4. 


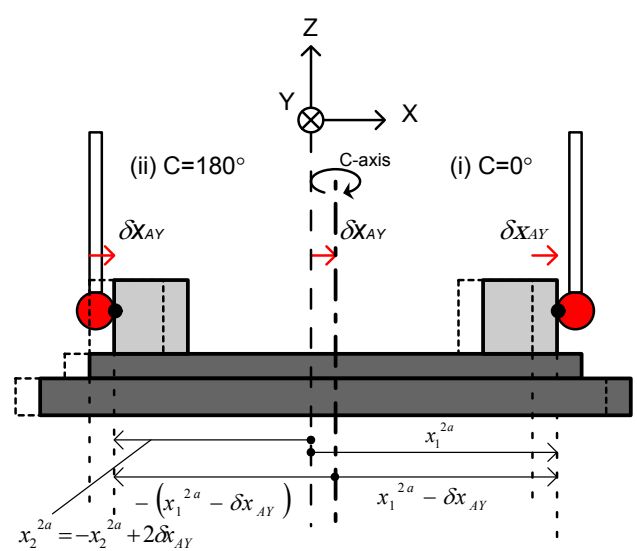

(a) Influence of $\delta x_{A Y}$.

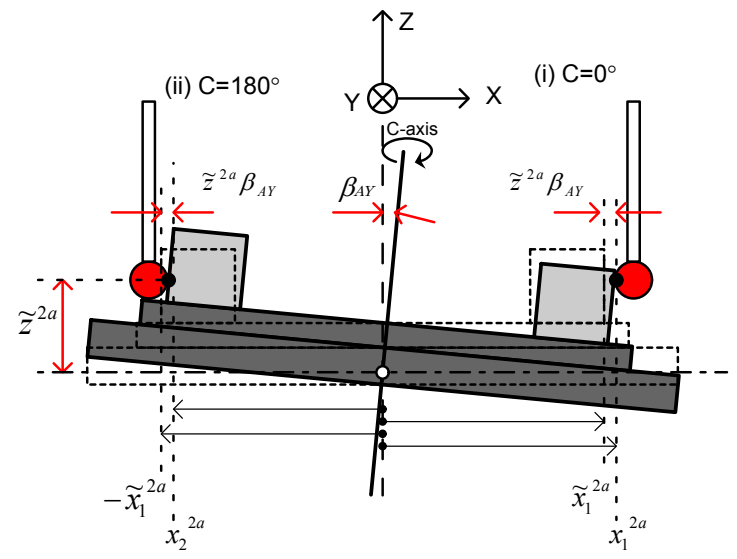

(b) Influence of $\beta_{A Y}$.

Figure 3: The influence of $\mathrm{C}$-axis location errors on probed positions. (a) Influence of $\delta x_{A Y}$, (b) Influence of $\beta_{A Y}$. 


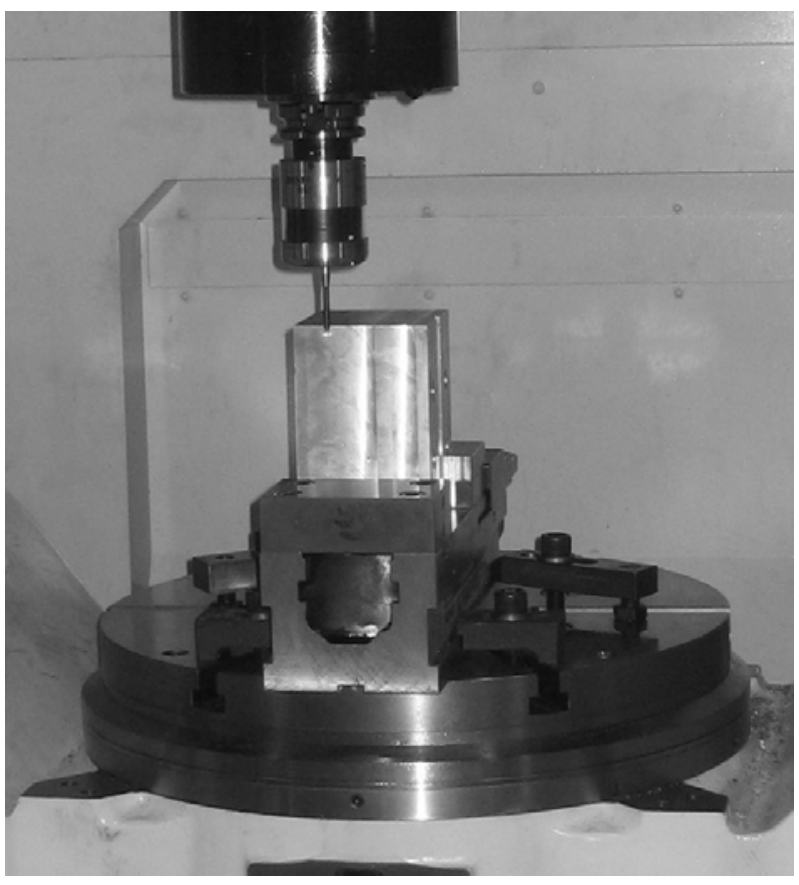

Figure 4: Experimental setup. 


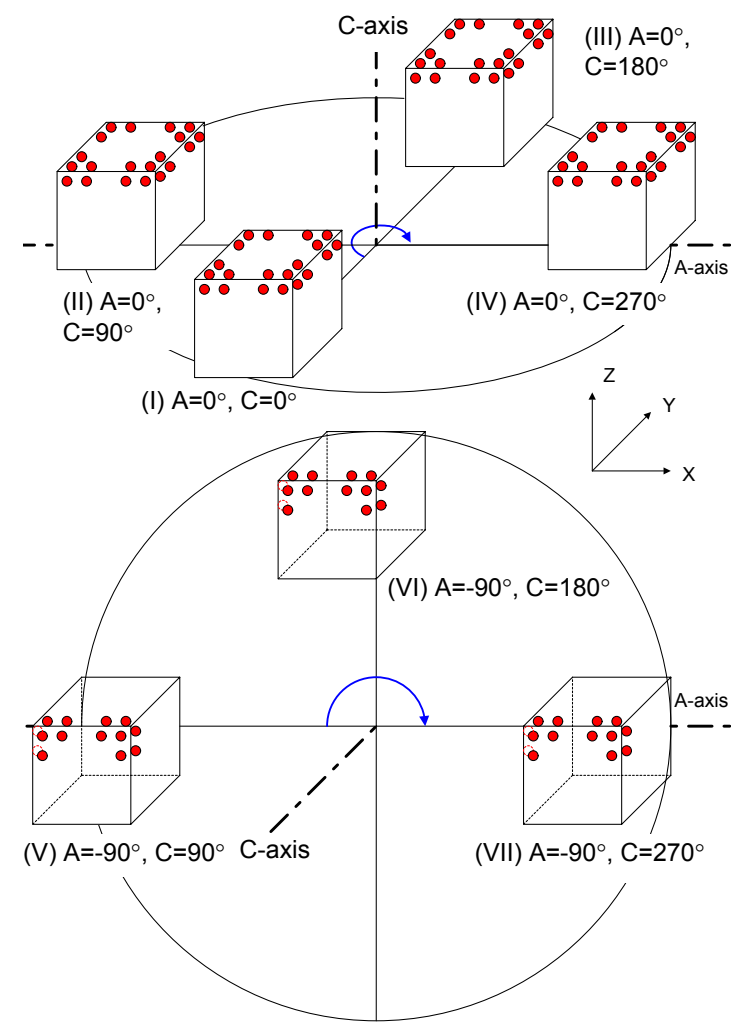

Figure 5: Probed points at each index angle of $\mathrm{A}$ and $\mathrm{C}$ axes. 


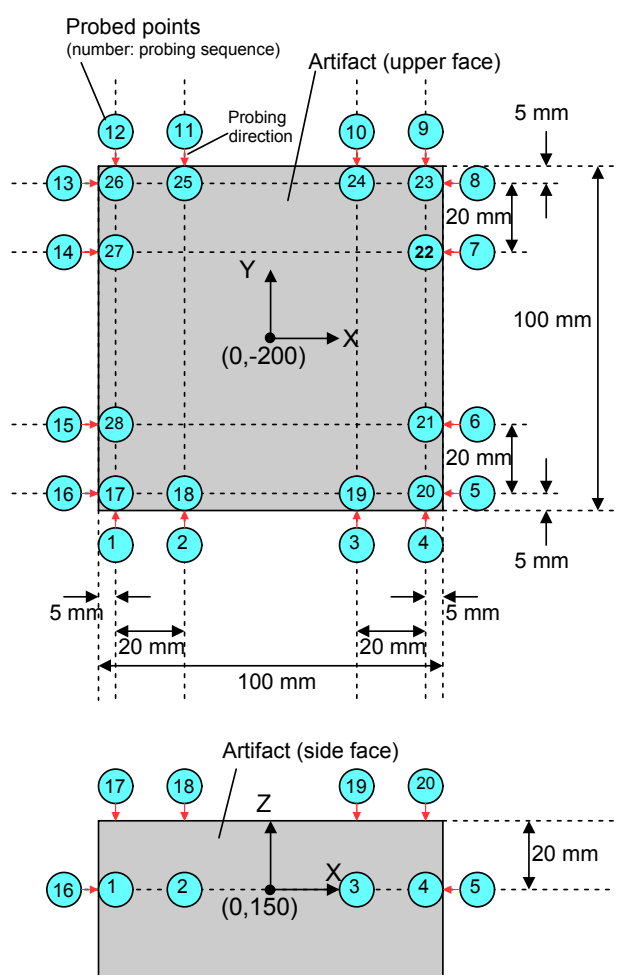

Figure 6: Position of probed points and probing sequence in the workpiece coordinate system. 


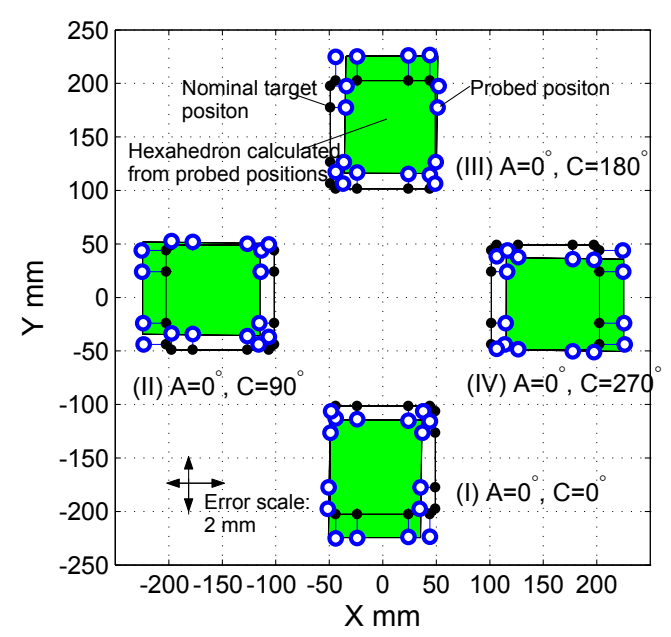

(a) Projection onto XY plane.
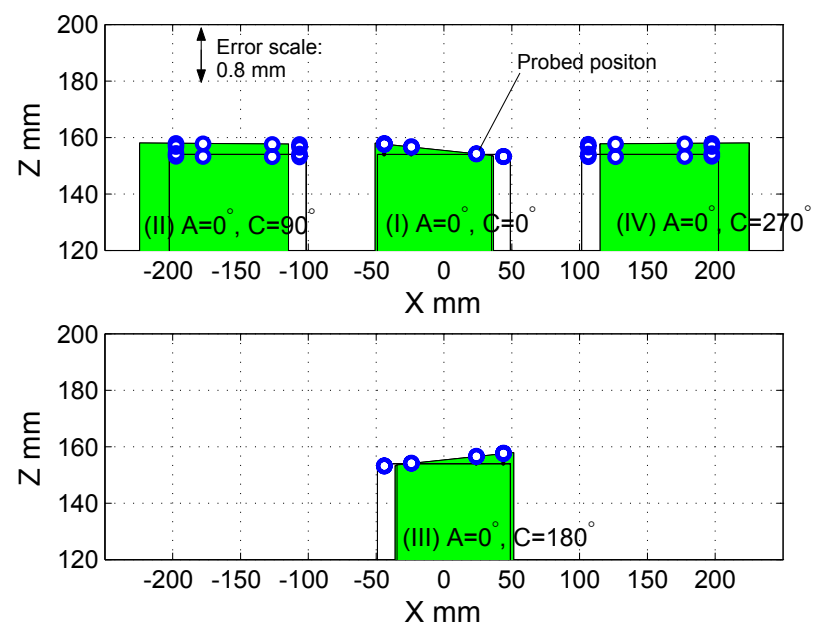

(b) Projection onto XZ plane.

Figure 7: "Raw" probed positions for (I) to (IV) $\left(A=0^{\circ}, C=0,90,180,270^{\circ}\right)$. Errors are magnified 25 times. (a) Projection onto XY plane. (b) Projection onto XZ plane. 


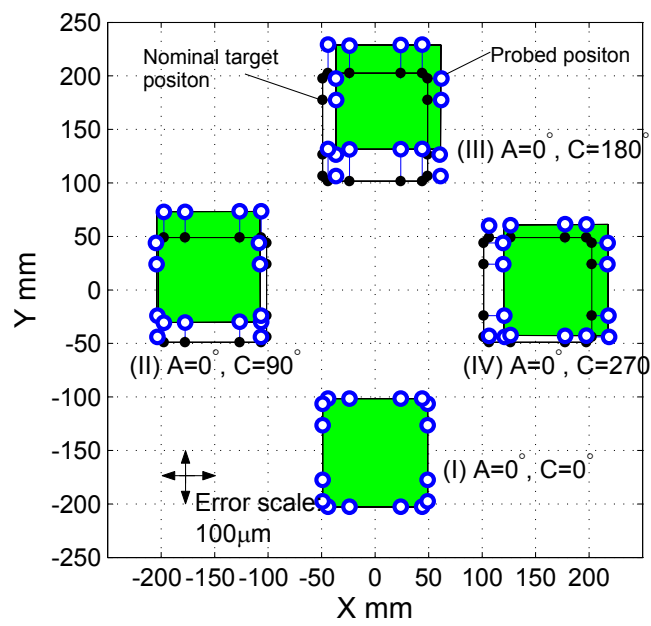

(a) Projection onto XY plane.
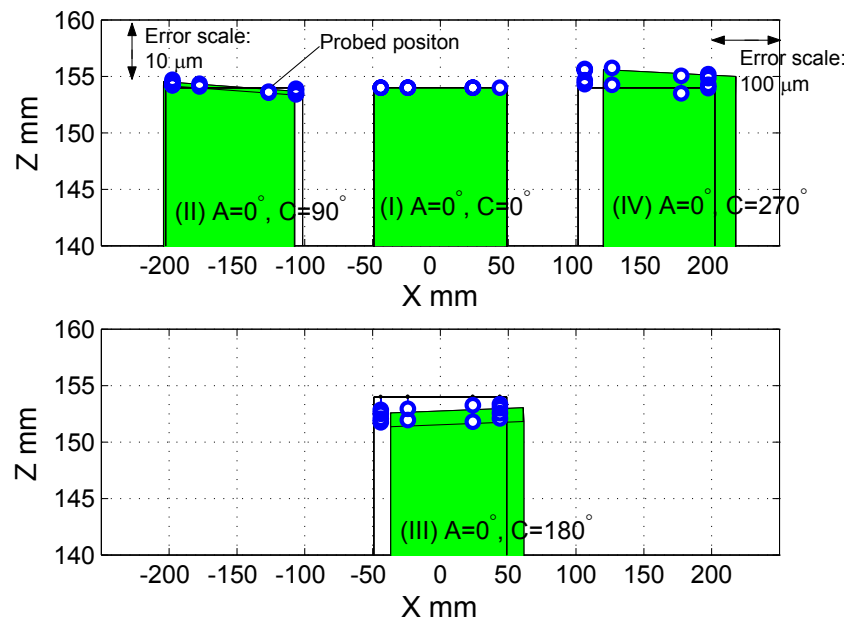

(b) Projection onto XZ plane. 

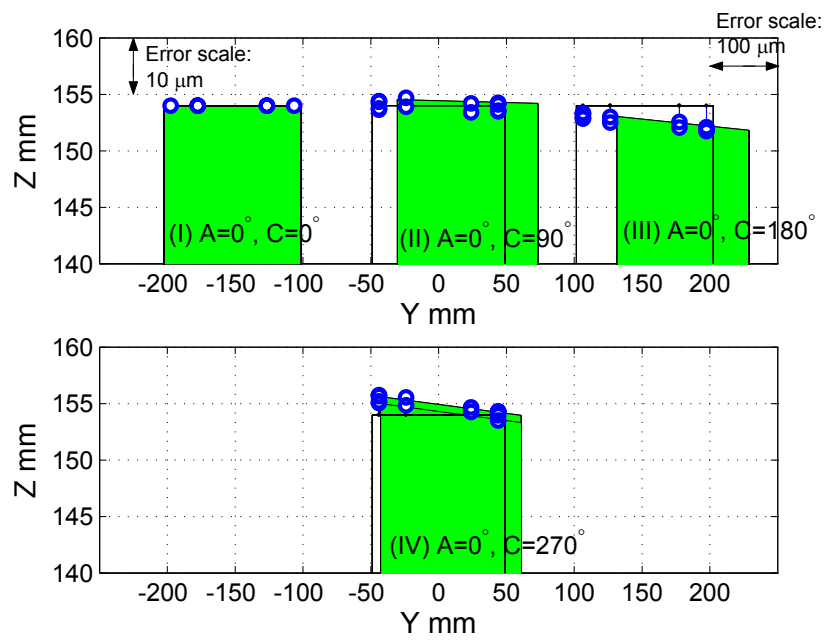

(c) Projection onto YZ plane.

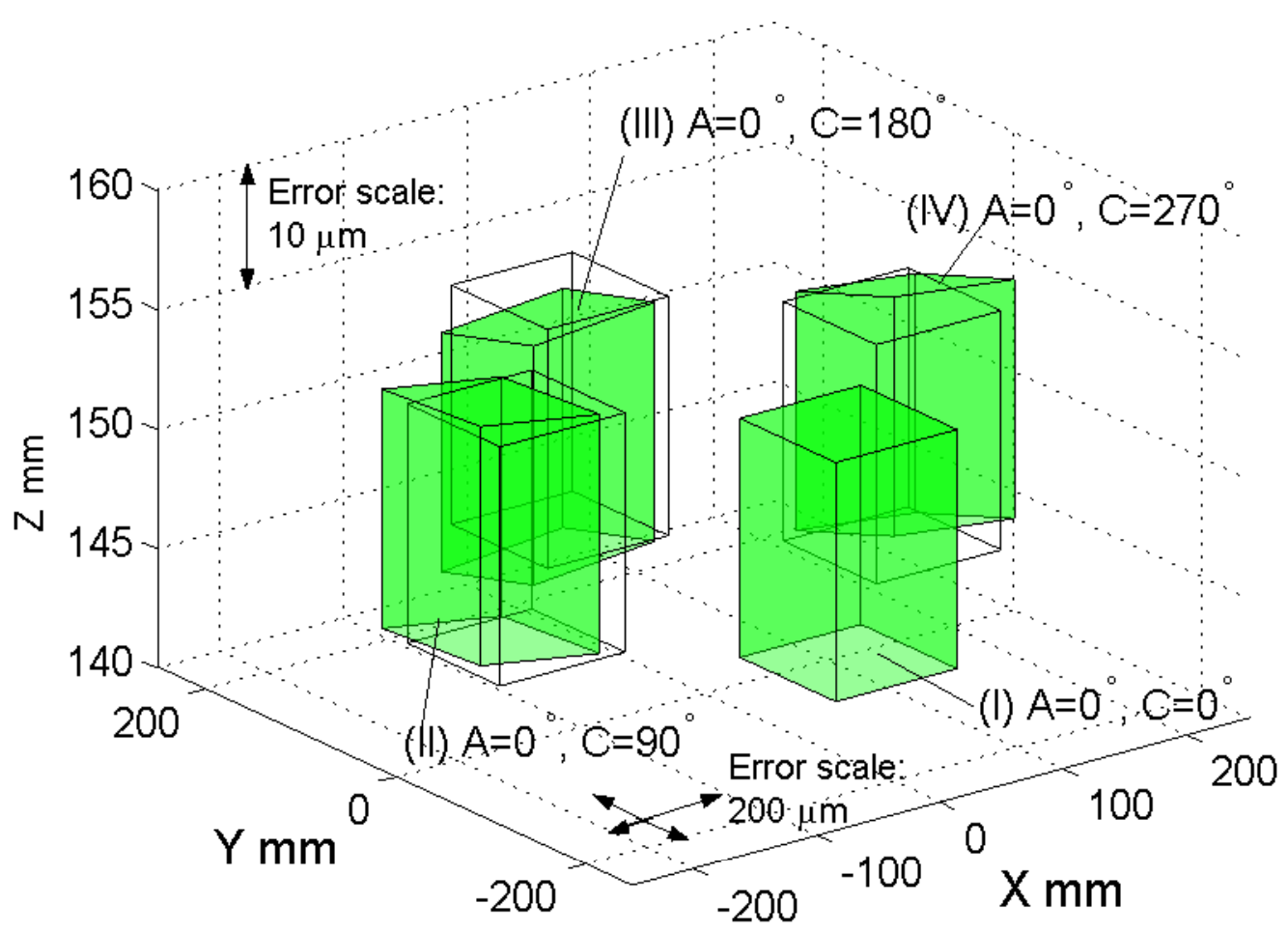

(d) In three-dimensional view (only average planes of probed points are shown).

Figure 8: Probed positions for (II) to (IV) $\left(A=0^{\circ}, C=90,180,270^{\circ}\right)$ in relative to corresponding points in (I) $\left(C=0^{\circ}\right)$. Errors are magnified 500 times. (a) Projection onto XY plane. (b) Projection onto XZ plane, (c) Projection onto YZ plane, (d) In three-dimensional view (only average planes of probed points are shown). 


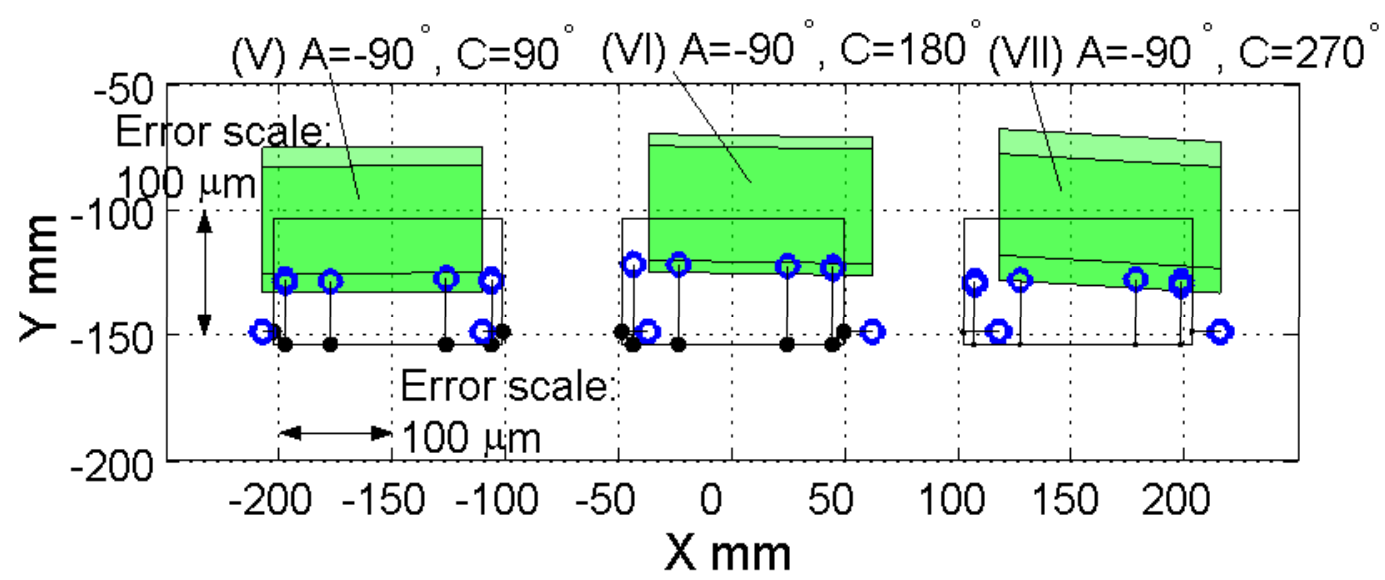

(a) Projection onto XY plane.

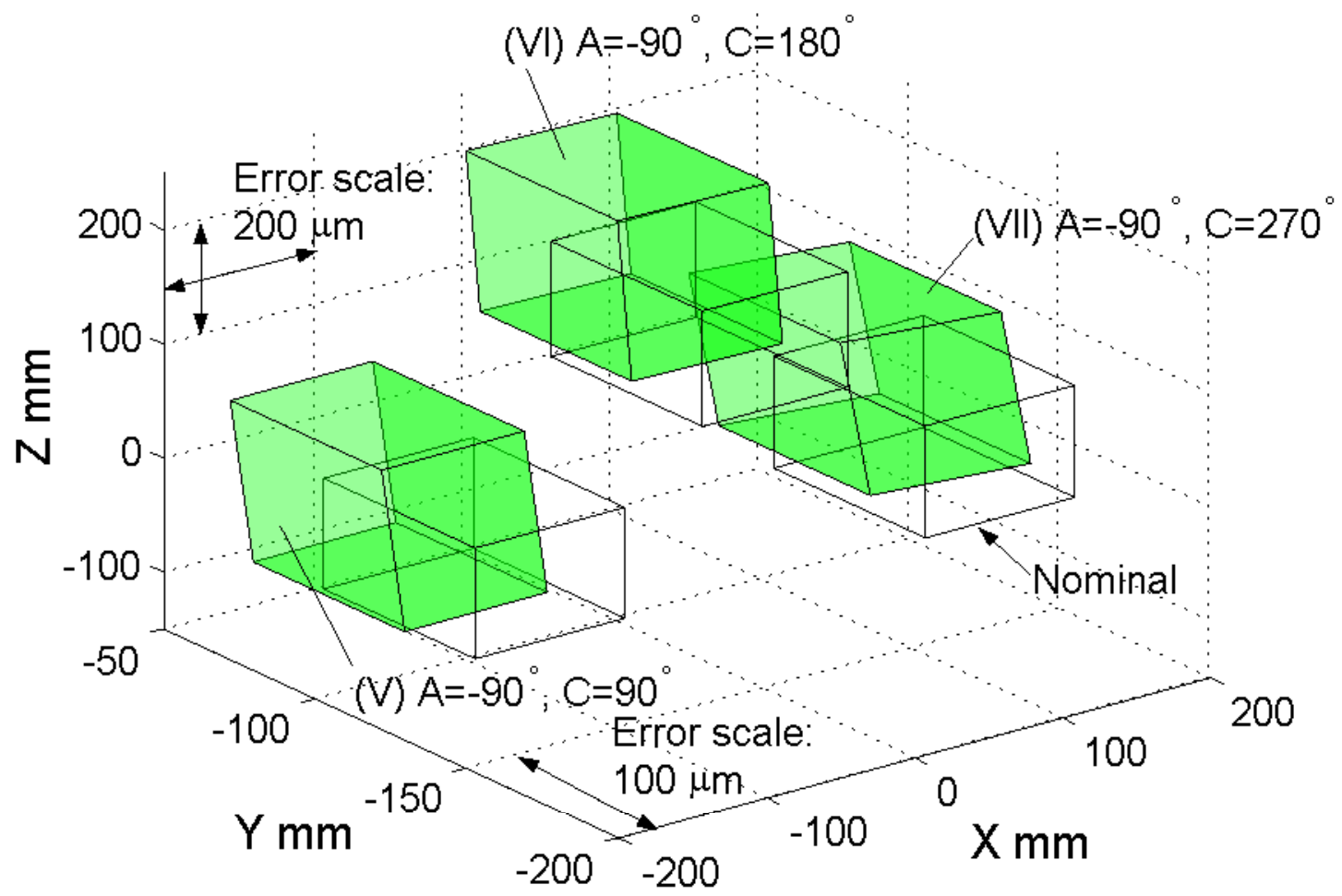

(b) In three-dimensional view (only 49 verage planes of probed points are shown).

Figure 9: Probed positions for (V) to (VII) $\left(A=-90^{\circ}, C=90,180,270^{\circ}\right)$ in relative to corresponding points in (I) $\left(A=0^{\circ}, C=0^{\circ}\right)$. Errors are magnified 500 times. (a) Projection onto XY plane. (b) In three-dimensional view (only average planes of probed points are shown). 